See discussions, stats, and author profiles for this publication at: https://www.researchgate.net/publication/230741635

\title{
Segmentation of the Common Carotid Intima-Media Complex in Ultrasound Images Using Active Contours
}

Article in IEEE transactions on bio-medical engineering · August 2012

DOI: 10.1109/TBME.2012.2214387 . Source: PubMed

CITATIONS

29

4 authors:

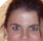

Styliani Petroudi

University of Cyprus

55 PUBLICATIONS 448 CITATIONS

SEE PROFILE

Marios Pantzaris

Cyprus Institute of Neurology and Genetics

176 PUBLICATIONS 2,112 CITATIONS

SEE PROFILE

\section{READS}

140

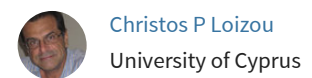

155 PUBLICATIONS 1,811 CITATIONS

SEE PROFILE

C. S. Pattichis

University of Cyprus

431 PUBLICATIONS 5,663 CITATIONS

SEE PROFILE

Some of the authors of this publication are also working on these related projects:

Linked 2 Safety View project

GRANATUM "A social collaborative working space semantically interlinking biomedical researchers knowledge and data for the design and execution of in-silico models

and experiments in cancer chemoprevention" FP7 Project View project 


\title{
Segmentation of the Common Carotid Intima-Media Complex in Ultrasound Images Using Active Contours
}

\author{
Styliani Petroudi*, Christos Loizou, Marios Pantziaris, and Constantinos Pattichis
}

\begin{abstract}
The segmentation of the intima-media complex (IMC) of the common carotid artery (CCA) wall is important for the evaluation of the intima media thickness (IMT) on B-mode ultrasound (US) images. The IMT is considered an important marker in the evaluation of the risk for the development of atherosclerosis. The fully automated segmentation algorithm presented in this article is based on active contours and active contours without edges and incorporates anatomical information to achieve accurate segmentation. The level set formulation by Chan and Vese using random initialization provides a segmentation of the CCA US images into different distinct regions, one of which corresponds to the carotid wall region below the lumen and includes the far wall IMC. The segmented regions are used to automatically achieve image normalization, which is followed by speckle removal. The resulting smoothed lumen-intima boundary combined with anatomical information provide an excellent initialization for parametric active contours that provide the final IMC segmentation. The algorithm is extensively evaluated on $\mathbf{1 0 0}$ different cases with ground truth (GT) segmentation available from two expert clinicians. The GT mean IMT value is $0.6679 \mathrm{~mm}+/-0.1350 \mathrm{~mm}$ and the corresponding automatically segmented (AS) mean IMT value is $0.6054 \mathrm{~mm}$ $+/-0.1464 \mathrm{~mm}$. The mean absolute difference between the GT IMT and the IMT evaluated from from the AS region is $0.095 \mathrm{~mm}$ $+/-0.0615 \mathrm{~mm}$. The polyline distance is $0.096 \mathrm{~mm}+/-0.034 \mathrm{~mm}$ while the Hausdorff distance is $0.176 \mathrm{~mm}+/-0.047 \mathrm{~mm}$. The algorithm compares favorably to both automatic and semiautomatic methods presented in the literature.
\end{abstract}

Index Terms-Active contours, carotid artery, intima-media thickness, level sets, segmentation, ultrasound imaging.

\section{INTRODUCTION}

$\mathbf{C}$ ARDIOVASCULAR disease (CVD) is one of the most common causes of death in the western world and stroke is the most common cause of disability in women. Therefore, the need to identify the asymptomatic patients at higher risk is great. The main pathophysiological mechanism leading to CVD is the

Manuscript received November 5, 2011; revised May 29, 2012; February 17, 2012; accepted July 12, 2012. Date of publication August 21, 2012; date of current version October 16, 2012. Asterisk indicates corresponding author.

${ }^{*}$ S. Petroudi is with the Department of Computer Science, University of Cyprus, Nicosia 1678, Cyprus (e-mail: styliani@ucy.ac.cy).

C. Loizou is with the Department of Computer Science, Intercollege, Limassol 51604, Cyprus (e-mail: loizou.c@lim.intercollege.ac.cy).

M. Pantziaris is with the Cyprus Institute of Neurology and Genetics, Nicosia 23462, Cyprus (e-mail: pantziari@ cing.ac.cy).

C. Pattichis is with the Department of Computer Science, University of Cyprus, Nicosia 1678, Cyprus (e-mail: pattichi@ucy.ac.cy).

Color versions of one or more of the figures in this paper are available online at http://ieeexplore.ieee.org.

Digital Object Identifier 10.1109/TBME.2012.2214387 development of atherosclerosis: the degeneration of the arterial walls though lipid and other blood-borne material on vascular territories throughout the body. Carotid intima media thickness (IMT), which is the distance between the lumen-intima and the media-adventitia interfaces, is a measure of early atherosclerosis. It can be evaluated quantitatively, noninvasively and with low cost, using high-resolution B-mode ultrasound (US), where it can be seen as the double line pattern on both walls of the longitudinal images of the common carotid artery (CCA) [1].

Carotid IMT is correlated with all traditional vascular risk factors and is regarded as an intermediate phenotype of atherosclerosis or a marker of subclinical organ damage. It provides an index of individual atherosclerosis and has been shown to positively correlate with the severity of atherosclerosis and predict cardiovascular events independent of traditional risk factors [2]. Even though other measures such as carotid wall irregularity [3] can be used for the diagnosis of atherosclerosis, IMT is the measure most commonly used at present in clinical practice. Thus, the IMT may be used for the screening of population as at least half of premature heart attacks and strokes, can, and should, be prevented [4]. IMT can be measured through the segmentation of the intima media complex (IMC), which corresponds to the intima and media layers of the arterial wall. Proper evaluation of the carotid IMT could be used to identify people at higher risk than traditional CVD risk factors alone [5]. The determination of the IMC boundaries is, however, a complicated task, as the IMC is a thin, relatively low contrast structure, that may be affected by US artifacts, may appear differently due to either different imaging angles and/or differences in anatomy, and deteriorates with age [6].

There is a number of techniques that have been proposed for the segmentation of the IMC [7]-[11]; but, most of them are semiautomatic and need user intervention. Different methods have been developed for carotid artery segmentation using gradient- and edge-based techniques [7], [9], dynamic programming techniques [8], texture and other image features [12], parametric active contours-based segmentations [10], [13], Hough transform segmentation [14], as well as combinations thereof. A review of some of the state of the art algorithms for IMT evaluation is presented in [15].

More recently Rocha et al. [16] presented an integrated method that uses edge detection and curve fitting to establish the adventitia boundary. Dynamic programming along with intensity thresholding are used to establish the lumen boundary and geometric active contours are used to smooth the lumen outline. Mahmoud et al. [17] developed a semiautomatic 
TABLE I

OVERVIEW OF RECENT REPRESENTATIVE CCA IMC SEGMENTATION TECHNIQUES FOR US IMAGING BY METHOD

\begin{tabular}{|c|c|c|c|c|c|c|}
\hline Study & Year & Common Carotid Artery IMC Segmentation Technique & UI & $\begin{array}{l}\text { Evaluation } \\
\text { Method }\end{array}$ & $I M T_{\text {error }}(\mathrm{mm})$ & $\mathrm{N}$ \\
\hline $\begin{array}{l}\text { Stein et al. } \\
{[11]}\end{array}$ & 2005 & $\begin{array}{l}\text { Edge based method using intensity and gradient information com- } \\
\text { bined with morphological smoothing for inter and intra observer } \\
\text { variability evaluation }\end{array}$ & Yes & $\begin{array}{l}\text { Mean } \\
\text { distance }\end{array}$ & $0.04+/-0.007$ & $50 / 300$ \\
\hline $\begin{array}{l}\text { Mahmoud et } \\
\text { al. [17] }\end{array}$ & 2010 & $\begin{array}{l}\text { Edge based method using a multi-step coarse to fine gradient } \\
\text { based method that incorporates intensities, gradients and pixel } \\
\text { continuity }\end{array}$ & Yes & $\begin{array}{l}\text { Mean } \\
\text { Distance }\end{array}$ & $0.016+/-0.0387$ & 45 \\
\hline $\begin{array}{l}\text { Liang et al. } \\
{[8]}\end{array}$ & 2000 & $\begin{array}{l}\text { Multi-scale dynamic programming for finding a global optimum } \\
\text { cost function, incorporating different image and geometrical fea- } \\
\text { tures }\end{array}$ & $\mathrm{MC}$ & $\begin{array}{l}\text { Mean } \\
\text { Distance }\end{array}$ & 0.042 & 50 \\
\hline $\begin{array}{l}\text { Cheng et al. } \\
{[21]}\end{array}$ & 2010 & $\begin{array}{l}\text { Edge based method combining gradient evaluation (MacLeod } \\
\text { Filter) followed by feature enhancement combined with dynamic } \\
\text { programming that incorporates additional anatomical information }\end{array}$ & Yes & $\begin{array}{l}\text { Mean } \\
\text { Distance }\end{array}$ & 0.1154 & $\begin{array}{l}275 \text { im- } \\
\text { ages }\end{array}$ \\
\hline $\begin{array}{l}\text { Meiburger et } \\
\text { al. [20] }\end{array}$ & 2011 & $\begin{array}{l}\text { Scale-space multi-resolution analysis that involves speckle fil- } \\
\text { tering and smoothing followed by mean shift clustering for } \\
\text { estimation of the density gradient and boundary reconstruction }\end{array}$ & No & $\begin{array}{l}\text { Mean } \\
\text { Distance } \\
\text { PD }\end{array}$ & $0.039+/-0.186$ & $\begin{array}{l}300 \mathrm{im}- \\
\text { ages }\end{array}$ \\
\hline Xu et al. [19] & 2011 & $\begin{array}{l}\text { Edge analysis provides the input for Hough transform on contin- } \\
\text { uous image segments where Dual Snakes provide the final LI and } \\
\text { MA outlines }\end{array}$ & Yes & $\begin{array}{l}\text { Mean } \\
\text { distance }\end{array}$ & $0.038+/-0.0164$ & $\begin{array}{l}50 \\
\text { images }\end{array}$ \\
\hline $\begin{array}{l}\text { Rocha et al. } \\
{[16]}\end{array}$ & 2010 & $\begin{array}{l}\text { Spline fitting on evaluated edges, followed by dynamic program- } \\
\text { ming where the final segmentation is smoothed using geometric } \\
\text { active contours }\end{array}$ & Yes & $\begin{array}{l}\text { Mean } \\
\text { distance }\end{array}$ & $0.07+/-0.11$ & $24 / 47$ \\
\hline $\begin{array}{l}\text { Molinari et } \\
\text { al. [18] }\end{array}$ & 2011 & $\begin{array}{l}\text { Inter-greedy technique fusing different IM segmentation methods } \\
\text { including signal processing, snakes, and watershed segmentation }\end{array}$ & No & $\begin{array}{l}\text { Mean } \\
\text { distance }\end{array}$ & $0.0463+/-0.469$ & 200 \\
\hline
\end{tabular}

AIC automatic initial contour, UI user interaction, MC manual correction possible, $\mathrm{N}$ number of cases/images, LI lumen intima, MA media adventitia, PD polyline distance.

method that uses gradient information to evaluate the IMT. In the manually selected region of interest, a Gaussian blurring filter is used to remove noise followed by the column-wise evaluation of gradient to establish the intima media boundaries. Continuity and energy constraints are used to establish the correct outlines. Molinari et al. [18] took the IMT segmentation results from three IMT segmentation methods: 1) signal processing approach, combined with snakes and fuzzy clustering, 2) integrated approach based on seed and line detection, followed by probability-based connectivity and classification, and 3) morphological approach, with watershed transform and fitting, and fused the resulting boundaries using a greedy method described by the "ball and basket" to minimize the system error. Xu et al. [19] used the Hough transform to establish line segments corresponding to the IMT and segment the image to vertical segments for evaluating the possible outlines. The resulting boundaries are used to initialize dual snakes which, in turn, are used for imposing stricter shape deformation constraints. Meiburger et al. [20] used a two step fully automatic algorithm that first identified the lumen using multiresolution analysis and then segmented the IMC using the mean shift algorithm. However, further extensive postprocessing is required to remove erroneous objects, establish which outlines correspond to the interfaces and finally obtain and smooth the final profiles. Cheng et al. [21] developed a semiautomatic method, where the selected region was filtered for gradient extraction and enhancement. Dual dynamic programming was used to find the lumen-intima and media-adventitia layers that incorporate anatomical information such as possible anatomy segmentation thickness and geometry. Rocha et al. [22] presented a new semiautomatic method that used the traditional image processing using anisotropic diffusion-based edge detection followed by edge selection using dominant gradient direction and anatomi- cal characteristics. After edge selection, they used the Random Sample Consensus algorithm on cubic splines to find the best smooth global path corresponding to the IMC outlines. Some of the recent representative publications are summarized in Table I.

Different level set models and methodologies have been developed and used for the segmentation of US images in different clinical applications. Sarti et al. [23] developed a geometrybased model which uses a selected fixation point to built an initial surface based on the corresponding point-of-view, detects existing local features and evolves the surface using both the geometry of the surface and image features for finding the level set that best describes the object. They demonstrated the method on 3-D echocardiography and fetal echography. Angelini et al. [24] developed a region-based level set similar to the formulation by Chan and Vese [25] to segment endocardial surfaces on denoised US data. A review of the relevant literature is given in [26]. Other more recent methods, exploiting the Chan and Vese model for US processing and segmentation include [27], [28] and [29].

This study presents a fully automated algorithm for the segmentation of the far wall IMC. The algorithm uses active contours [30], and active contours without edges [25]. Active contours without edges [25] with the incorporation of anatomical information are used to establish intensity information for the US images normalization. Following image normalization, active contours without edges are again used to identify an initial intima-media boundary. This boundary provides an excellent, completely automatic initialization for the snake segmentation algorithm that gives the final IMC segmentation. The resulting fully automated segmentation is validated against two expert clinicians manual IMC segmentations and corresponding IMT measures. The following section presents the method in greater detail. Finally, the resulting segmentation and corresponding 
measures are presented and discussed. A preliminary version of this study has appeared in [31].

\section{METHOD}

The presented method is based on the use of active contours [30] and active contours without edges [25] to segment different regions in the carotid US images. Active contours [30] face limitations such as initialization, curve parameterization, and the inability to deal with images where the different structures have many components. The level set formulation of the active contours without edges by Chan and Vese [25] represents curves in an implicit manner, and can handle changes in topology [32], which has been one of the main difficulties that snake-based CCA algorithms faced [10], [13]. The active contours without edges algorithm [25] is used to segment the US images into the lumen and the carotid wall, and the segmentation results are combined with anatomical information to evaluate the needed parameters and process the US image for accurate segmentation of the IMC at different stages of the algorithm.

\section{A. Characteristics of US Longitudinal CCA Images}

For the evaluation of the IMT, B-mode longitudinal US images of the CCA are used, which display the vascular wall as a regular pattern that correlates with anatomical layers. The images cover longitudinally the carotid artery and show the near wall, the lumen and the far wall as shown in Fig. 1. IMT appears as a double-line pattern on both walls of the CCA in the longitudinal US image, and consists of the leading edges of two anatomical boundaries: the lumen-intima and media-adventitia. Anatomical and tissue characteristics and differences result in the lumen appearing as a large longitudinal passage between two brighter regions. The adventitia layer is echogenic and in turn appears as much brighter. The presented algorithm explores these anatomical characteristic differences of the CCA, to achieve the segmentation of the far wall IMC and the evaluation of the IMT.

\section{B. Segmentation Using Active Contours Without Edges and Image Normalization}

A fully automated IMC segmentation algorithm needs to be able to work on all images despite variability due to capture time, settings, and scanners. The normalization of B-mode US images for the CCA has been shown to address this variability using intensity adjustments that take into account anatomy and differences in tissue attenuation. The normalization method proposed in [33] performs linear grayscale remapping so that median intensity value of the artery lumen has intensities between 0 and 5 , and the median intensity value of the adventitia between 180 and 190 for 8 bit US images [34]. The normalization reduces image variability due to the aforementioned reasons. However, the normalization in [33] requires human interaction for choosing a region in the lumen and another in the adventitia so that corresponding intensity values are established for the intensity remapping. To find the intensity values for the application of image normalization the lumen and the adventitia need to be
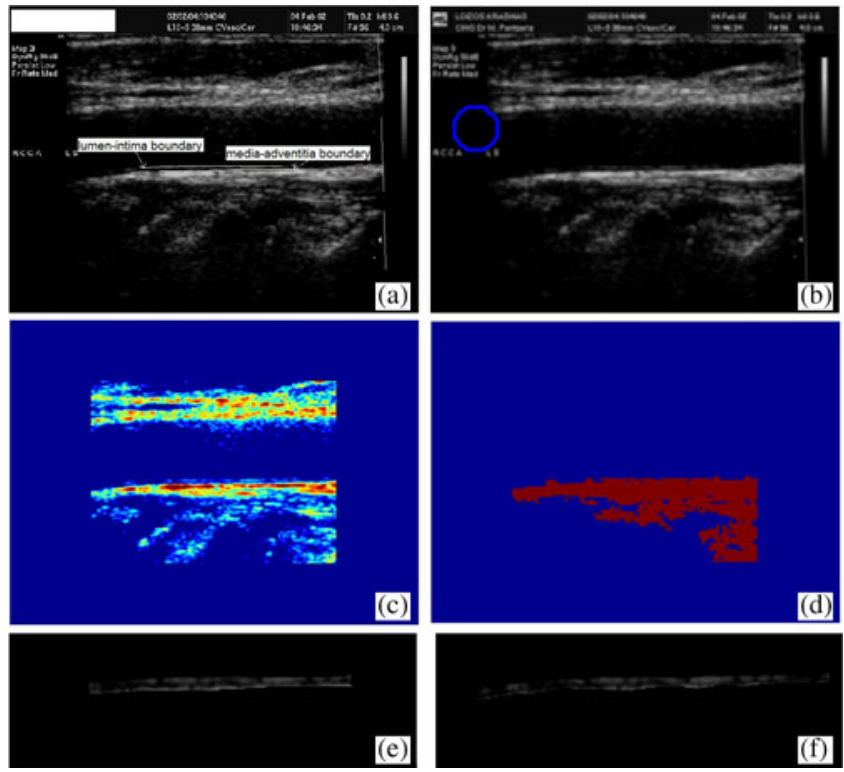

Fig. 1. Level set segmentation of CCA image. (a) US image with the IMC GT outline superimposed. (b) Level set initialization. (c) Segmentation of the US image in different regions. (d) Segmented far wall intima-media-adventitia. (e) GT IMC. (f) AS IMC.

segmented. This segmentation is achieved with the application of level sets.

Level set methods [32] offer a highly robust and accurate method for tracking interfaces moving under complex motions: they work in a number of space dimensions but more importantly they can handle topological changes naturally. Using the level set formulation of the active contours without edges by Chan and Vese [25], the regions corresponding to the lumen and the carotid wall (including the intima, the media and the adventitia) are automatically segmented (AS), as shown in Fig. 1(b) and (c).

The Chan-Vese model [25] corresponds to a region-based level set method which uses the Mumford-Shah functional in the level set framework for a piecewise constant representation of an image. The evolution of the curve is governed by properties of the region of the image $u_{0}(x, y)$ enclosed by the curve. The model tries to separate the image into regions based on pixel intensities and introduces the following energy functional:

$$
\begin{aligned}
F\left(c_{1}, c_{2}, C\right)= & \mu \cdot \operatorname{Length}(C)+\nu \cdot \operatorname{Area}(\operatorname{inside}(C)) \\
& +\lambda_{1} \int_{\operatorname{inside}(C)}\left|u_{0}(x, y)-c_{1}\right|^{2} d x d y \\
& +\lambda_{2} \int_{\text {outside }(C)}\left|u_{0}(x, y)-c_{2}\right|^{2} d x d y
\end{aligned}
$$

where $\mu \geq 0, \nu \geq 0, \lambda_{1}, \lambda_{2}>0$, are fixed parameters. Equation (5) is a generalization of the Mumford-Shah functional as introduced in [35]. Segmentation becomes the minimization problem of

$$
\inf _{c_{1}, c_{2}, C} F\left(c_{1}, c_{2}, C\right)
$$


where $c_{1}$ is the mean of $u_{0}$ inside the curve $C$, and $c_{2}$ is the mean of $u_{0}$ outside the curve $C$. The parameters $\lambda_{1}, \lambda_{2}, \nu$, and $\mu$ are parameters selected to fit the particular class of images. This minimal partition problem is formulated using level sets [32]. In the level set method [32], the curve $C$ defined in $\Omega$ is represented by the zero level set of a Lipschitz function $\phi: \Omega \rightarrow \mathbb{R}$ such that

$$
\left\{\begin{array}{l}
C=\partial \Omega=\{(x, y) \in \Omega: \phi(x, y)=0\} \\
\operatorname{inside}(C)=\Omega=\{(x, y) \in \Omega: \phi(x, y)>0\} \\
\text { outside }(C)=\Omega \backslash \bar{\omega}=\{(x, y) \in \Omega: \phi(x, y)<0\} .
\end{array}\right.
$$

Using the Heaviside function $H$ and the 1-D Dirac measure $\delta_{0}$, defined, respectively, by

$$
H(z)= \begin{cases}1 & \text { if } z \geq 0 \\ 0 & \text { if } z<0\end{cases}
$$

and

$$
\delta_{0}=\frac{d}{d z} H(z)
$$

and replacing the unknown variable $C$ by the unknown variable $\phi$ the energy functional $F$ can be rewritten as follows:

$$
\begin{aligned}
F\left(c_{1}, c_{2}, \phi\right)= & \mu \cdot \int_{\Omega} \delta(\phi(x, y))|\nabla \phi(x, y)| d x d y \\
& +\nu \int_{\Omega} H(\phi(x, y)) d x d y \\
& +\lambda_{1} \int_{\Omega}\left|u_{0}(x, y)-c_{1}\right|^{2} H(\phi(x, y)) d x d y \\
& +\lambda_{2} \int_{\Omega}\left|u_{0}(x, y)-c_{2}\right|^{2}(1-H(\phi(x, y))) d x d y .
\end{aligned}
$$

Minimization is achieved as presented by Chan and Vese [25]. Without loss of generality the initialization contour is a disk with radius of $2.1 \mathrm{~mm}$ randomly placed in the image background (segmented from simple thresholding with a threshold close to zero). For the evaluation of the level set segmentation in addition to downsampling the original US image by a factor of 4 to accelerate the segmentation, the value of the different parameters used are: $\mu=0.2$ and $h$ the space step is set to 1 while $\Delta t$ the time step, is set to 0.25 . Also, for computation of the regularized versions of $H$ and $\delta_{0} \varepsilon=1$ as used by [25]. The values for the different parameters for the Chan-Vese model were empirically verified. The empirical verification also showed that slight variation in the value of the parameters would not have a significant effect on the final segmentation. The first two terms in (5) control the regularity by penalizing the length and the enclosed area of $\mathrm{C}$. The value of $\mu$ is a penalty on the total length of the edge contour for a given segmentation, which provides a balance between fitting the image more accurately (smaller $\mu$ ) and resulting in a smoother boundary the larger its value the smoother the boundary (larger $\mu$ ). The choice of $\mu=0.2$ allows the segmentation to accurately match the data, while still resulting in a relatively smooth curve. The term $\nu$ provides a penalty on the foreground area resulting from the segmentation and here is set to 0 . The terms $\lambda_{1}$ and $\lambda_{2}$ weight region uniformity penalizing the discrepancy between the piecewise constant model $u$ and the input image. For segmenting the lumen and the adventitia, the energy contributed by the intensity variance of the two regions is equally weighted to $\lambda_{1}=\lambda_{2}=1$. In a large number of applications [25], including this implementation $\lambda_{1}=\lambda_{2}=1$ and $\nu=0$. However, parameters of the energy functional may need to be modified if new smoothness constraints are needed or if data sets with different visual quality are used [24].

The method is robust to speckle as well as irregular contrast, but most importantly it is completely automated, does not require user interaction and is not depended on edges. After the Chan-Vese model is applied the image is segmented into a number of regions some corresponding to the artery wall while others to the background and the lumen. More importantly, the resulting segmentation can also serve as an excellent initialization for the parametric active contours for the segmentation of the IMC. For establishing the adventitia and the lumen and without loss of generality the areas corresponding to the first decile in the left and the last decile in the right are set to zero intensity, as are the first decile on the top and the last on the bottom. This way, the patient information, the machine settings as well as other graphical markings placed during acquisition are removed and the image is constrained to only contain the imaged anatomy as shown in Fig. 1(c). Thus, the resulting image, after the application of the Chan-Vese consists mainly of large regions corresponding to the near and far walls of the CCA with a darker region between them corresponding to the lumen. Following, only the regions with an area larger than $18 \mathrm{~mm}^{2}$ are considered so that the main regions corresponding to the near and far walls are investigated and used for normalization [13] and very small regions are removed. By running lines vertically across the middle of the segmented and restricted US image and looking at the corresponding intensity profiles, the largest region on the bottom of the image, which results from the segmentation using the Chan-Vese model followed by the aforementioned postprocessing, corresponds to the far carotid wall, while the large darker/background region above it corresponds to the lumen. This is the region of interest for evaluating the values for image normalization as well as for the initialization of the snake. By evaluating the intensities in the corresponding regions' areas using mean filtering, image normalization as described in [36] can be automatically achieved. The maximum mean intensity value of the far wall (adventitia) and the minimum mean intensity value of the lumen (blood) are used as reference points to linearly scale the image so that the median gray level value of the blood is 0 and the median gray value of the adventitia is 190, in the same way the US images are linearly scaled in [13].

Fig. 1 shows the initialization of the Chan-Vese segmentation and results of the Chan-Vese algorithm. It can be seen that the largest region in the bottom of Fig. 1(c) corresponds to the far wall three layers (intima-media-adventitia) of the CCA. The segmentation of this region provides the required intensity information to achieve image normalization. More importantly, the boundary of this region provides the outline for the initialization of the parametric active contour segmentation, as described in the following section. 


\section{Speckle Removal}

Speckle is a form of granular multiplicative noise caused when the surface imaged appears rough to the scale of the wavelength used. Speckle noise has a significant impact on the correctness of boundary detection. The edges of the adventitia are also affected by this noise. Thus, prior to any other filtering, the speckle removal linear scaling filter that utilizes local mean and variance, as in [33], is applied to despeckle the image.

\section{Segmentation of the IMC Using Active Contours}

The boundary of the far wall adventitia, resulting from the application of the Chan-Vese level set [25] provides an excellent initialization for the segmentation of the IMC using snakes [30], as in [13]. The extracted boundary, between the lumen and the adventitia approximates very closely the lumen-intima boundary and provides the contour points needed to run the snake. The gradient of the line that best fits the initialization boundary, using least squares error approximation, provides a constraint for the choice of points used to initialize the parametric active contour. Twenty equally spaced contour points, with a local gradient that is within $5 \%$ of the lumen-intima line approximation's gradient, are chosen along the identified boundary. These points are matched with 20 points which are displaced $0.9 \mathrm{~mm}$ downward in the vertical direction. This displacement is based on the observation that the IMT lies between 0.6 and $1.4 \mathrm{~mm}$, with a mean IMT of $1.0 \mathrm{~mm}$ [37]. Unlike [13], the initialization points are equally spaced. Prior to the segmentation using parametric active contours, directional smoothing is applied, using a simple averaging filter of $0.12 \mathrm{~mm} \times 0.3 \mathrm{~mm}$ in the longitudinal direction that aids in the discrimination of the media and helps reduce the attraction by other edges.

The segmentation of the IMC is achieved as in [13], minimizing the active contour energy functional [30] using the fast algorithm extension by Williams and Shah [38]:

$$
\begin{aligned}
E_{\text {snake }}^{*}= & \int_{0}^{1} E_{\text {snake }}(\mathbf{v}(s)) d s \\
= & \int_{0}^{1}\left(E_{\text {internal }}(\mathbf{v}(s))+E_{\text {image }}(\mathbf{v}(s))\right. \\
& \left.+E_{\text {constraints }}(\mathbf{v}(s))\right) d s
\end{aligned}
$$

where $\mathbf{v}(s)=(x(s), y(s))$ is the vector representation of the contour with the arc length $s$ as the parameter. $E_{\text {internal }}$ corresponds to the internal regularization energy that imposes continuity and bending constraints and comprises two components, while $E_{\text {image }}$ represents the image energy incorporating local gradient magnitude:

$E=\int\left\{\frac{1}{2} \alpha(s)\left|\mathbf{v}^{\prime}(s)\right|^{2}+\frac{1}{2} \beta(s)\left|\mathbf{v}^{\prime \prime}(s)\right|^{2}+\gamma(s)\left(-|\nabla I(\mathbf{v})|^{2}\right)\right\} d s$

where prime denotes differentiation and the parameters $\alpha, \beta$, and $\gamma$ are normalizing factors used to balance the relative influence of the three energy term. For initialization of the snake deformation and the minimization of (7), the following initial values are used: $\alpha_{i}(s)=0.6, \beta_{i}(s)=0.4$, and $\gamma_{i}(s)=2$, as in [10] and [13]. The resulting segmentation corresponds to the IMC.

\section{E. Segmentation Evaluation Methods}

The segmentation of the far wall IMC is evaluated using three different measures: mean absolute distance, Hausdorff distance [39], and polyline distance (PD) [40]; and several corresponding statistics are presented based on the comparison of the continuous outlines of the intima and the media resulting from the presented method. Let AS correspond to the boundary of the IMC resulting from the automatic segmentation, and let GT correspond to the ground truth boundary of the IMC taken as the mean of the manual delineations provided by the expert clinicians as is done in other works [15].

1) Mean Absolute Distance: Mean absolute distance $D_{\mid \text {mean } \mid}$ is simply the mean value of the absolute Euclidean distance differences between the AS boundary and the GT. It is defined as follows:

$$
D_{\mid \text {mean } \mid}(\mathrm{AS}, \mathrm{GT})=\frac{1}{N} \sum_{y y=1}^{N}|\mathrm{AS}(y y)-\mathrm{GT}(y y)|
$$

where $y y$ is an index corresponding to the image columns and $N$ is the total number of columns in the segmented/outlined IMC, as used in [13] and [41]. Other studies present the signed mean distance [42]. $D_{|\operatorname{mean}|}$ provides a good approximation of the error especially when the anatomy of the IMC segmented region is mostly straight and horizontal but also tends to overestimate the error otherwise. In addition to $D_{\mid \text {mean }}$, the Bland-Altman plot [43] are also presented to enable comparison with more methods in the literature [15].

2) Hausdorff Distance: The Hausdorff distance $H(\mathrm{AS}, \mathrm{GT})$ is the maximum distance of a set to the nearest point in the other set. The Hausdorff distance between the two sets of points can be defined as follows:

$$
H(\mathrm{AS}, \mathrm{GT})=\max _{a s \in \mathrm{AS}}\left\{\min _{g t \in \mathrm{GT}}\{d(a s, g t)\}\right\}
$$

where $a s$ and $g t$ are each a point from sets AS and GT, respectively, and $d(a s, g t)$ is any metric - here the Euclidean distance is used between these points. Through this definition one can say that any point of AS is at most at distance $H(\mathrm{AS}, \mathrm{GT})$ to some point of GT. Since the Hausdorff distance is oriented the more symmetric general definition:

$$
H(\mathrm{AS}, \mathrm{GT})=\max \{H(\mathrm{AS}, \mathrm{GT}), H(\mathrm{GT}, \mathrm{AS})\}
$$

is used. Thus, the Hausdorff distance provides an upper limit on the distance between the points in the AS and the GT boundaries as used by Destrempes et al. [41].

\section{F. Polyline Distance}

The PD between two polygons, here the AS and the GT, is defined as the average distance between a vertex of one polygon and the boundary of another [40] or as the closest distance between each estimated boundary point to the GT boundary. The basic idea is to measure the distance of each vertex of a boundary to the segments of the other boundary. Following [40], 
let $d(v, s)$ correspond to the distance between a point $v$ and a line segment $s$. Then

$$
d(v, s)= \begin{cases}\min \left(d_{1}, d_{2}\right), & \text { if } \lambda<0, \lambda>1 \\ \left|d^{\perp}\right| ; & \text { if } 0 \leq \lambda \leq 1\end{cases}
$$

where $d_{1}, d_{2}$ are the distances from the vertex $v$ to the end points of the corresponding segment $s$ and $d^{\perp}$ is the perpendicular distance from the vertex $v$ to $s$ and $\lambda$ is the distance along $s$.

The PD from vertex $v$ to the GT segmentation boundary is defined as follows:

$$
d_{b}(v, \mathrm{GT})=\min _{s \in \operatorname{sides} \mathrm{GT}} d(v, s)
$$

and identifies the closest segment from $v$ to GT and the distance between the automatically extracted AS polygon and the sides of the ground truth GT IMC segmented boundaries is calculated by:

$$
d_{v b}(\mathrm{AS}, \mathrm{GT})=\sum_{v \in \text { verticesAS }} d(v, \mathrm{GT})
$$

To achieve a symmetrical result the PD measure used for the evaluation of the distance between the two segmented areas $\mathrm{PD}_{s}(\mathrm{AS}, \mathrm{GT})$ is defined by

$$
\mathrm{PD}_{s}(\mathrm{AS}, \mathrm{GT})=\frac{d_{v b}(\mathrm{AS}, \mathrm{GT})+d_{v b}(\mathrm{GT}, \mathrm{AS})}{\sharp \text { vertices } \in \mathrm{AS}+\sharp \text { vertices } \in \mathrm{GT}}
$$

which reflects the average distance between the two boundaries.

\section{RESULTS}

The algorithm is evaluated on longitudinal B-mode US images of the CCA obtained from 100 normal asymptomatic subjects acquired by the ATL HDI-3000 US scanner (Advanced Technology Laboratories, Seattle, WA), with a $7 \mathrm{MHz}$ $38 \mathrm{~mm}$ linear array transducer with an axial system resolution of $0.11 \mathrm{~mm}$. The 8-bit images i.e., with intensity ranging from 0 to 255 were resized using the bicubic method to have resolution of 16.66 pixels $/ \mathrm{mm}$ i.e., the spatial resolution is $0.06 \mathrm{~mm}$ and a corresponding size of $768 \times 576$ pixels in order to maintain uniformity in the digital image spatial resolution [13]. Two expert vascular clinicians (referred to as Ex1 and Ex2) delineated the IMT on the US images by defining about 20 consecutive points on the IMT border. The expert clinicians manually segmented all images, providing the delineation of the lumen-intima and the media-adventitia of the far carotid wall. For the delineations, it should be noted that the lumen-intima frontiers are more visible in the far wall, and thus the corresponding regions are used for AS evaluation.

Figs. 1 and 2 show the results of the segmentation of the IMC using the presented method and compare the results to the GT segmentations. By observing the difference between the two segmentations one can see how closely the algorithm approximates the clinician's evaluation. The method successfully segments the IMC as the IMT of the AS and the GT differ at most $0.2483 \mathrm{~mm}$. The successful detection of the lumenintima boundary as well as the media-adventitia boundary was achieved in all 100 cases. According to the American Society of Echocardiography the measurement of IMT involves tracing

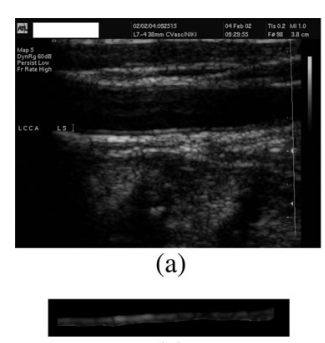

(c)

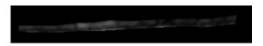

(b)

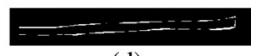

(d)
Fig. 2. Another example of the application of the presented IMC segmentation algorithm: (a) Original CCA US image. (b) Expert's GT segmentation. (c) Automated segmentation that results from the application of the presented algorithm. (d) Difference between the GT segmentation and the automated segmentation.

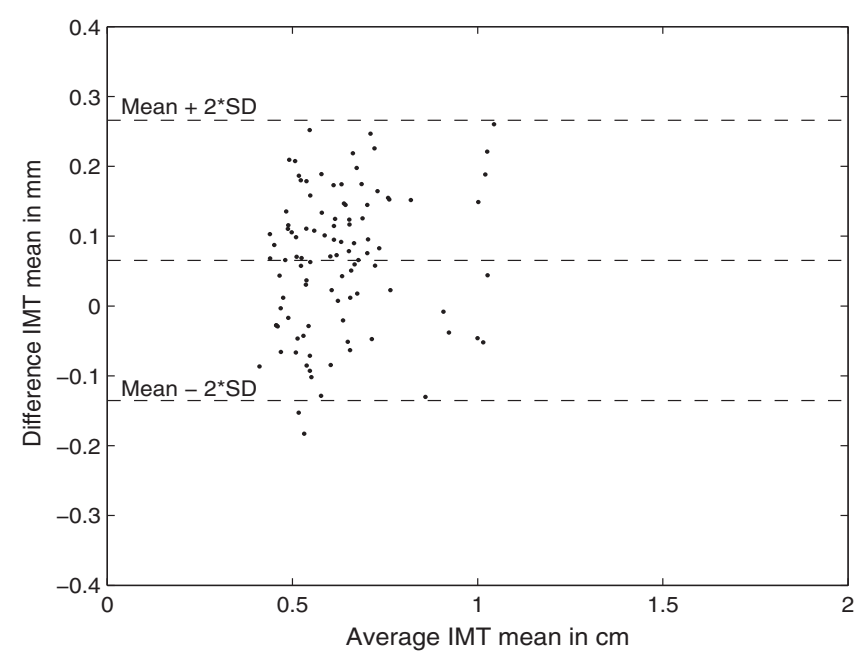

Fig. 3. Bland-Altman plot of the mean IMT for the GT and the AS in mm. SD corresponds to the standard deviation.

TABLE II

IMT VALUES AND STATISTICS FOR THE CAROTID ARTERY OF THE FAR WALL AND COMPARISON WITH [13]

\begin{tabular}{|l|c|c|c|c|}
\hline Intima Media Thickness in $\mathrm{mm}$ & Mean & Std & Min & Max \\
\hline AS & 0.6054 & 0.1464 & 0.3677 & 1.1746 \\
\hline GT & 0.6679 & 0.1350 & 0.4083 & 1.1812 \\
\hline Loizou et al. [13] & 0.68 & 0.12 & 0.49 & 0.87 \\
\hline Ex1 & 0.6495 & 0.1554 & 0.3977 & 1.2735 \\
\hline Ex2 & 0.6863 & 0.1644 & 0.4015 & 1.089 \\
\hline
\end{tabular}

the blood-intima and media-adventitia interfaces of the far wall and evaluating the corresponding mean IMT values from the far wall [5]. The mean value of the IMT for AS is $0.6054 \mathrm{~mm}$ while the mean value of the IMT for GT is $0.6678 \mathrm{~mm}$, a $0.0624 \mathrm{~mm}$ difference which corresponds to a $9.4 \%$ of the GT IMT-and at the images' given resolution to a difference of a pixel. The mean IMT values and other statistical information of automatic segmentation are presented in Table II. The mean value of the absolute differences between the AS and the GT, as well as the two experts' delineations are also presented in Table III. The mean absolute difference between the AS and the GT evaluation of the IMT corresponds to a $14 \%$ percentage difference. Fig. 3 shows the Bland-Altman plot of the difference between GT and the AS segmentations where the standard deviation is represented by SD. 
TABLE III

EVALUATION OF THE ABSOLUTE IMT DIFFERENCE BETWEEN THE AS VERSUS THE GT AND THE INDIVIDUAL EXPERT'S ANNOTATION FOR THE IMC SEGMENTATION

\begin{tabular}{|l|c|c|c|c|}
\hline Absolute IMT difference in $\mathrm{mm}$ & Mean & Std & Min & Max \\
\hline AS vs GT & 0.0950 & 0.0615 & 0.0007 & 0.2483 \\
\hline AS vs Ex1 & 0.1283 & 0.0756 & 0.0045 & 0.2972 \\
\hline AS vs Ex2 & 0.1013 & 0.0633 & 0.0032 & 0.2603 \\
\hline Ex1 vs Ex2 & 0.1003 & 0.0818 & 0.2349 & 0.3830 \\
\hline
\end{tabular}

\section{TABLE IV}

Evaluation OF THE PD MEASURE BETWEEN THE AS VERSUS THE GT AND THE INDIVIDUAL EXPERT'S ANNOTATION FOR THE SEGMENTATION OF THE FAR WALL IMC

\begin{tabular}{|ll|c|c|c|c|}
\hline Polyline Distance in mm & & Mean & Std & Min & Max \\
\hline AS vs GT & Intima & 0.096 & 0.034 & 0.038 & 0.162 \\
& Media & 0.057 & 0.029 & 0.019 & 0.111 \\
& $I M C$ & 0.076 & 0.021 & 0.049 & 0.125 \\
\hline AS vs Ex1 & Intima & 0.131 & 0.053 & 0.051 & 0.266 \\
& Media & 0.065 & 0.035 & 0.023 & 0.134 \\
& $I M C$ & 0.098 & 0.033 & 0.037 & 0.158 \\
\hline AS vs Ex2 & Intima & 0.067 & 0.034 & 0.018 & 0.159 \\
& Media & 0.082 & 0.024 & 0.034 & 0.138 \\
& $I M C$ & 0.075 & 0.023 & 0.026 & 0.136 \\
\hline Ex1 vs Ex2 & Intima & 0.1239 & 0.0537 & 0.0552 & 0.2242 \\
& Media & 0.0853 & 0.0507 & 0.00748 & 0.1957 \\
& $I M C$ & 0.1046 & 0.0459 & 0.0544 & 0.1891 \\
\hline
\end{tabular}

TABLE V

Evaluation of the HausdorfF Distance Measure Between the AS VERSUS THE GT AND THE INDIVIDUAL EXPERT'S ANNOTATION FOR THE SEGMENTATION OF THE FAR END IMC

\begin{tabular}{|ll|c|c|c|c|}
\hline Hausdorff Distance in mm & & Mean & Std & Min & Max \\
\hline AS vs GT & Intima & 0.176 & 0.047 & 0.090 & 0.300 \\
& Media & 0.143 & 0.065 & 0.060 & 0.335 \\
& $I M C$ & 0.190 & 0.050 & 0.150 & 0.335 \\
\hline AS vs Ex1 & Intima & 0.231 & 0.067 & 0.120 & 0.360 \\
& Media & 0.162 & 0.078 & 0.060 & 0.360 \\
& $I M C$ & 0.244 & 0.074 & 0.120 & 0.360 \\
\hline AS vs Ex2 & Intima & 0.208 & 0.097 & 0.060 & 0.540 \\
& Media & 0.227 & 0.096 & 0.060 & 0.569 \\
& $I M C$ & 0.258 & 0.107 & 0.085 & 0.569 \\
\hline Ex1 vs Ex2 & Intima & 0.234 & 0.083 & 0.12 & 0.42 \\
& Media & 0.217 & 0.093 & 0.060 & 0.436 \\
& $I M C$ & 0.251 & 0.086 & 0.18 & 0.436 \\
\hline
\end{tabular}

Tables IV and V present statistical information regarding the evaluated polyline and Hausdorff distance, for the upper and lower boundaries as well as the entire segmentation in the form of box-plots. The PD measure for the comparison of the AS and the GT is evaluated as the average of the PD for the lumen-intima boundary and the media-adventitia boundary as by definition the polyline measure is an average distance between a vertex of one boundary to the other [40]. On the other hand, the Hausdorff distance for the automatic segmentation of the IMC is evaluated as the maximum of the Hausdorff distances for the lumen-intima and the intima-media boundaries, a more strict error measure compared to the average, as the Hausdorff distance is the maximum distance of the one boundary to the nearest point in the other boundary.

Additionally, interreader analysis is also performed for all the presented measures were the measurements of Expert 1 are compared to those of Expert 2. The interobserver variability expressed as mean absolute difference for the measurement of common carotid IMT [44] was slightly higher than the mean absolute IMT difference between the presented fully automatic method and the GT, reaching 15\% of the GT IMT. From the corresponding values, one can see that the presented segmentation of the IMC and evaluation of the IMT consistently outperforms both the experts versus the GT and each other.

The algorithm was implemented using MATLAB (MathWorks Inc., Natick, MA), on an INTEL(R) $2.5 \mathrm{GHz}$ computer equipped with $6.00 \mathrm{~GB}$ of RAM, with an average processing time of $21 \mathrm{~s}$ making the method close to real time.

\section{DISCUSSION}

The presented method was developed to achieve accurate segmentation of the IMC and estimation of the IMT that closely approximate the clinician's evaluation without any user interaction. The method uses Chan-Vese level set segmentation [25] to get a good initial segmentation of the carotid artery wall, to automatically normalize the images and to provide a good initialization for the parametric active contours method which provide the final segmentation. The Chan-Vese segmentation was preferred over other methods such as the Hough transform [14] or the watershed segmentation [45] because it required no additional preprocessing to enhance edges, establish appropriate boundary lines and merge segmented regions. The algorithm is evaluated on a dataset of 100 cases, is robust to noise and considers different features at different levels. The geometric active contour initialization is automatic, does not need to be near the carotid artery and can capture the far wall IMC even when the US images do not capture the IMC but the carotid artery after it has branched in the internal and external carotid artery.

The evaluated mean IMT compares favorably to the GT estimation of the IMT as the mean values estimated for the AS and the GT segmentation differ by $0.0625 \mathrm{~mm}, 9.35 \%$ of the true value. However, when the absolute mean distance measure is evaluated, the difference between the two can be seen more distinctively, as both positive and negative differences are taken into account and the actual absolute difference is evaluated to $0.095 \mathrm{~mm}$. This can also be seen from the Bland-Altman plot. In addition, the results for the AS display a slightly higher dispersion, compared to the GT, but this can be attributed to images with poorer quality. Yet, the dispersion is still lower when compared to the individual clinicians' delineations and the corresponding interreader variability. However, at this point, it should be noted that the axial resolution of the US machine used to get the images used is $0.11 \mathrm{~mm}$ which means that if the IMT is thinner than $0.11 \mathrm{~mm}$ the two echo surfaces cannot be separated. In the same way, the final digital resolution of $0.06 \mathrm{~mm}$ resulting from interpolation means that there is an inherernt maximal deviation of 1 pixel, corresponding to $0.06 \mathrm{~mm}$ in 1 pair of measurement points [44]. The corresponding standard error can be reduced if multiple measurements are taken; however, this is based on the underlying assumption that the population follows a normal distribution and the measurements/samples are independent. Thus, the achieved accuracy and the corresponding interreader variability as seen in Table III show that the 
presented method may be used for aiding the clinician and for reducing interobserver variability.

The PD and the Hausdorff distance are evaluated separately for the lumen-intima and the media-adventitia boundaries and the corresponding estimations show similar variability for each boundary as well as for the delineation of the entire segmentation.

The Bland-Altman plot, shown in Fig. 3, comparing the mean IMT of the GT to the AS shows good agreement between the two segmentations with the mean difference close to $0.06 \mathrm{~mm}$ while in all but two cases the difference is within Bland-Altman's plot limits of agreement. It can also be seen that there is a large spread in the data.

Different results have been reported in the literature, ranging from a mean IMT distance error of $0.01 \mathrm{~mm}+/-0.0387 \mathrm{~mm}$ [17], which corresponds to 0.166 pixels to a mean IMT distance error of $0.1154 \mathrm{~mm}$ [21] which corresponds to almost 2 pixels, in terms of the images included in these studies. The differences can be attributed to the use of different datasets of different quality and size, the error measures, the availability of GT by more than one experts, and last but not least user interaction or manual user correction. In addition, only a number of studies provide absolute mean error values.

Mahmoud et al. [17] presented a mean error of $0.01 \mathrm{~mm}+/-$ $0.0387 \mathrm{~mm}$ in a database consisting of 45 cases from a method that however included user intervention.

Xu et al. [19] also developed a semiautomatic method which they evaluated on 50 images. The authors presented the mean absolute error for the lumen-intima boundary as $0.029 \mathrm{~mm}$ $+/-0.0230 \mathrm{~mm}$ and the mean absolute error for the mediaadventitia boundary as $0.049 \mathrm{~mm}+/-0.0245 \mathrm{~mm}$. The total of the two errors, corresponded to $0.078 \mathrm{~mm}$ and provided an upper boundary for the total IMT error.

Meiburger et al. [20] evaluated their fully automated method on a database of 300 cases, using the PD. The method achieved a bias (mean PD error) of $0.039 \mathrm{~mm}+/-0.186 \mathrm{~mm}$. The PD error between the lumen-intima was evaluated at $0.0499 \mathrm{~mm}$ and the media-adventitia boundary at $0.414 \mathrm{~mm}$. Additionally, even though the Bland-Altman plot between the GT and the automatically evaluated IMT is centered around almost $0 \mathrm{~mm}$, the difference values were almost normally distributed between -0.4 and $0.4 \mathrm{~mm}$.

Stein et al. [11] used the mean distance to evaluate their semiautomatic algorithm for the segmentation of the IMC, which resulted in a bias of $0.012 \mathrm{~mm}+/-0.0006 \mathrm{~mm}$. The corresponding values in the Bland-Altman plot showed that the errors are distributed between -0.05 and $0.1 \mathrm{~mm}$ with a few close to the estimated bias.

Rocha et al. [16] achieved good agreement between their segmentation and the GT through a semiautomatic method. They extensively evaluated their method and provided box plots for the mean distance for each boundary of the IMC. Again, the results of the method they developed were also presented in the Bland-Altman plots, with reported means ranging between -0.07 and $0.11 \mathrm{~mm}$.

The method presented here includes important improvements compared to [13]. The presented method is completely automated-there is no user interaction or manual correction. The normalization is achieved automatically after the application of the Chan-Vese level sets segments the lumen and the carotid wall. Additionally, the segmentation of the carotid wall provides an excellent lumen-intima initialization boundary for the William and Shah snake application. The good initialization boundary greatly enhances the ability of the method to converge to the correct IMC boundary. The two methods are evaluated on the same set of images. Compared to [13], the positioning of the initial snake contour was calculated correctly for all cases. The use of snakes ensures a continuous smooth boundary. However, the mean IMT value evaluated by the method in [13] as presented in Table II appears to be closer to the GT mean IMT than the method presented here, yet there is a much greater variation in the corresponding minimum and maximum values. That is why the Hausdorff distance-which can be thought of as the maximum mismatch-for the IMC segmentation is also investigated. The Hausdorff distance has greatly improved from $0.312 \mathrm{~mm}$ reported in [13] to $0.19 \mathrm{~mm}+/-0.05 \mathrm{~mm}$. The mean IMT for each case is evaluated along the entire length of the segmented boundary, so if at some points the thickness between the intima and the media is smaller than the true difference, and at other points it is greater than the true thickness the mean value can result being close to the true value. Thus, the Hausdorff distance provides a more reliable and concrete measure of the resemblance between the two segmentations as well as an upper boundary of the error/difference between the two outlines. The fact that the PD value is also very close to the mean absolute distance speaks to the robustness of the segmentation. The method is independent of equipment settings and no training is required.

The processing time of the procedure is relatively high $21 \mathrm{~s}$ compared to other methods [46] where an average processing time of $3 \mathrm{~s}$ is reported, as the segmentation is achieved using active contours, but is comparable with a number of other fully automated methods which require $30-50 \mathrm{~s}$ [15]. Still, the processing time is not prohibitive in the use of the presented method in a "quasi-real time" clinical setting, as manual clinical measurement times range between 7 and 18 min [44].

\section{CONCLUSION}

Measurement of IMT with B-mode US is a non-invasive and sensitive technique for identifying and quantifying subclinical vascular disease and for evaluating cardiovascular risk in clinical practice [5]. US images of the carotid artery pose a great challenge to automatic segmentation, due not only to their complexity but also to several factors that can introduce variability including operator dependence and acquisition settings. The presented segmentation of the IMC and evaluation of the IMT provide an objective and reproducible technique. The method is fully automatic, achieves lower absolute mean IMT error compared to interobserver variability in the evaluated dataset, has comparable results to both automatic and semiautomatic methods in the literature (Table 1) and takes into account instrumental variability through image normalization [13], [36]. The method may be used in clinical practice for the reliable segmentation of the carotid artery and the estimation of features of the arterial 
wall with physiological significance for the evaluation of early stages of atherosclerosis and corresponding risks. The presented technique may represent a generalized and standard methodology towards completely automated and accurate IMT measurement. It may be used for aiding the clinicians through providing support in their examination, reducing not only reading times, but also the variability between readers whilst improving reproducibility [5], [37]. It can be used for evaluating the IMT in large multicenter studies [37] for developing and evaluating risk models and screening methods. More importantly, future work will involve the use of the presented method for the evaluation of different features including wall irregularity and area texture measures, in addition to the IMT, for the establishment of risk models for the development of cardiovascular disease to aid the clinicians in screening and evaluation of treatment.

\section{ACKNOWLEDGMENT}

The authors would like to thank Prof. A. Nicolaides and Ms. N. Georgiou at the Cyprus Cardiovascular Educational and Research Trust for their contribution and for discussions regarding US carotid imaging.

\section{REFERENCES}

[1] P. Touboul, M. Hennerici, S. Meairs, H. Adams, P. Amarenco, N. Bornstein, L. Csiba, M. Desvarieux, S. Ebrahim, M. Fatar, R. Hernandez, M Jaff, S. Kownator, P. Prati, T. Rundek, M. Sitzer, U. Schminke, J. Tardif, A. Taylor, E. Vicaut, K. Woo, F. Zannad, and M. Zureik, "Mannheim carotid intima-media thickness consensus (2004-2006)," Cerebrovasc. Diseases, vol. 23, pp. 75-80, 2007, 2012.

[2] M. W. Lorenz, H. S. Markus, M. L. Bots, M. Rosvall, and M. Sitzer, "Prediction of clinical cardiovascular events with carotid intima-media thickness: A systematic review and meta-analysis," Circulation, vol. 115, no. 4, pp. 459-467, 2007.

[3] T. Ishizu, T. Ishimitsu, H. Kamiya, Y. Seo, N. Moriyama, K. Obara S. Watanabe, and I. Yamaguchi, "The correlation of irregularities in carotid arterial intima-media thickness with coronary artery disease," Heart Vessels, vol. 17, pp. 1-6, 2002.

[4] D. Lloyd-Jones, R. J. Adams, T. M. Brown, M. Carnethon, S. Dai, G. De Simone, T. Ferguson, E. Ford, K. Furie, C. Gillespie, A. Go, K. Greenlund, N. Haase, S. Hailpern, P. Ho, V. Howard, B. Kissela, S. Kittner, D. Lackland, L. Lisabeth, A. Marelli, M. McDermott, J. Meigs, D. Mozaffarian, M. Mussolino, G. Nichol, V. Roger, W. Rosamond, R. Sacco, P. Sorlie R. Stafford, T. Thom, S. Wasserthiel-Smoller, N. Wong, and J. WylieRosett, "Heart disease and stroke statistics 2010 update. A report from the american heart association," Circulation, vol. 121, no. 7, pp. 948-954, 2009.

[5] J. H. Stein, C. E. Korcarz, R. T. Hurst, E. Lonn, C. B. Kendall, E. Mohler, S. Najjar, C. Rembold, and W. Post, "Use of carotid ultrasound to identify subclinical vascular disease and evaluate cardiovascular disease risk: A consensus statement from the American Society of Echocardiography carotid intima-media thickness task force. Endorsed by the Society for Vascular Medicine," J. Amer. Soc. Echocardiogr., vol. 21, pp. 93-111, 2008.

[6] D. Lamont, L. Parker, M. White, N. Unwin, S. M. A. Bennett, M. Cohen, D. Richardson, H. O. Dickinson, A. Adamson, K. G. M. Alberti, and A. W. Craft, "Risk of cardiovascular disease measured by carotid intimamedia thickness at age 49-51: Lifecourse study," Br. Med. J., vol. 320, no. 7230, pp. 273-278, 2000.

[7] P. Pignoli and T. Longo, "Evaluation of atherosclerosis with b-mode ultrasound imaging," J. Nucl. Med. All. Sci., vol. 32, pp. 166-173, 1988.

[8] Q. Liang, I. Wendelhag, J. Wikstrand, and T. Gustavsson, "A multiscale dynamic programming procedure for boundary detection in ultrasonic artery images," IEEE Trans. Med. Imag., vol. 19, no. 2, pp. 127-142, Feb. 2000.

[9] R. H. Selzer, W. J. Mack, P. L. Lee, H. Kwong-Fu, and H. N. Hodis, "Improved common carotid elasticity and intima-media thickness mea- surements from computer analysis of sequential ultrasound frames," Atherosclerosis, vol. 154, no. 1, pp. 185-193, 2001.

[10] D. Cheng, A. Schmidt, K. Cheng, and H. Burkhardt, "Using snakes to detect the intimal and adventitial layers of the common carotid artery wall in sonographic images," Comput. Methods Progr. Biomed., vol. 67, no. 1, pp. 27-37, 2002.

[11] J. H. Stein, C. E. Korcarz, M. E. Mays, P. S. Douglas, M. Palta, H. Zhang, T. LeCaire, D. Paine, D. Gustafson, and L. Fan, "A semiautomated ultrasound border detection program that facilitates clinical measurement of ultrasound carotid intima-media thickness," J. Amer. Soc. Echocardiogr. vol. 18, no. 3, pp. 244-251, 2005.

[12] A. Mojsilovic, M. Popovic, N. Amodaj, R. Babic, and M. Ostojic, "Automatic segmentation of intravascular ultrasound images: A texture-based approach," Ann. Biomed. Eng., vol. 25, pp. 1059-1071, 1997.

[13] C. Loizou, C. Pattichis, M. Pantziaris, T. Tyllis, and A. Nicolaides, "Snakes based segmentation of the common carotid artery intima media," Med. Biol. Eng. Comput., vol. 45, pp. 35-49, 2007.

[14] S. Golemati, J. Stoitsis, E. G. Sifakis, T. Balkizas, and K. S. Nikita, "Using the hough transform to segment ultrasound images of longitudinal and transverse sections of the carotid artery," Ultrasound Med. Biol., vol. 33, no. 12, pp. 1918-1932, 2007.

[15] F. Molinari, G. Zeng, and J. S. Suri, "A state of the art review on intimamedia thickness (IMT) measurement and wall segmentation techniques for carotid ultrasound," Comput. Methods Progr. Biomed., vol. 100, pp. 201221, 2010.

[16] R. Rocha, A. Campilho, J. Silva, E. Azevedo, and R. Santos, "Segmentation of the carotid intima-media region in b-mode ultrasound images," Image Vis. Comput., vol. 28, pp. 614-625, 2010.

[17] A. Mahmoud, A. Morsy, and E. de Groot, "A new gradient-based algorithm for edge detection in ultrasonic carotid artery images," in Proc. Annu. Int. Conf. IEEE Eng. Med. Biol. Soc., 2010, pp. 5165-5168.

[18] F. Molinari, G. Zeng, and J. S. Suri, "Inter-greedy technique for fusion of different segmentation strategies leading to high-performance carotid IMT measurement in ultrasound images," Atheroscler. Disease Manag., New York: Springer, 2011, pp. 253-279.

[19] X. Xu, Y. Zhou, X. Cheng, E. Song, and G. Li, "Ultrasound intima-media segmentation using hough transform and dual snake model," Computerized Med. Imag. Graph., vol. 36, no. 3, pp. 248-258, 2012.

[20] K. M. Meiburger, F. Molinari, U. R. Acharya, L. Saba, P. Rodrigues, W. Liboni, A. Nicolaides, and J. S. Suri, "Automated carotid artery intima layer regional segmentation," Phys. Med. Biol., vol. 56, no. 13, pp. 4073 4090, 2011

[21] D.-C. Cheng, A. Schmidt-Trucksss, C.-H. Liu, S.-H. Liu, "Automated detection of the arterial inner walls of the common carotid artery based on dynamic b-mode signals," Sensors, vol. 10, no. 12, pp. 10601-10619, 2010 .

[22] R. Rocha, A. Campilho, J. Silva, E. Azevedo, and R. Santos, "Segmentation of ultrasound images of the carotid using ransac and cubic splines," Computer Methods Progr. Biomed., vol. 101, no. 1, pp. 94-106, 2011.

[23] A. Sarti, R. Malladi, and J. Sethian, "Subjective surfaces: A geometric model for boundary completion," Int. J. Comput. Vis., vol. 46, pp. 201221, 2002.

[24] E. D. Angelini, S. Homma, G. Pearson, J. W. Holmes, and A. F. Laine, "Segmentation of real-time three-dimensional ultrasound for quantification of ventricular function: A clinical study on right and left ventricles," Ultrasound Med. Biol., vol. 31, no. 9, pp. 1143-1158, 2005.

[25] T. Chan and L. Vese, "Active contours without edges," IEEE Trans. Image Process., vol. 10, no. 2, pp. 266-277, Feb. 2001.

[26] E. Angelini, J. Yinpeng, and A. Laine, "State of the art of level set methods in segmentation and registration of medical imaging modalities," Handbook of Biomedical Image Analysis, Registration Models, pp. 47-102, 2005

[27] G. Slabaugh, G. Unal, M. Wels, T. Fang, and B. Rao, "Statistical regionbased segmentation of ultrasound images," Ultrasound Med. Biol., vol. 35, no. 5, pp. 781-795, 2009.

[28] Q. Duan, E. D. Angelini, and A. F. Laine, "Real-time segmentation by active geometric functions," Comput. Methods Progr. Biomed., vol. 98, no. 3, pp. 223-230, 2010.

[29] D. Barbosa, T. Dietenbeck, J. Schaerer, J. D'hooge, D. Friboulet, and O. Bernard, "B-spline explicit active surfaces: An efficient framework for real-time 3-D region-based segmentation," IEEE Trans. Image Process., vol. 21 , no. 1, pp. 241-251, Jan. 2012.

[30] M. Kass, A. Witkin, and D. Terzopoulos, "Snakes: Active contour models," Int. J. Comput. Vis., vol. 1, pp. 321-331, 1988.

[31] S. Petroudi, C. Loizou, M. Pantziaris, M. Pattichis, and C. Pattichis, "A fully automated method using active contours for the evaluation of the 
intima-media thickness in carotid us images," in Proc. IEEE Int. Conf. Eng. Med. Biol., 2011, pp. 8053-8057.

[32] S. Osher and J. A. Sethian, "Fronts propagating with curvature-dependent speed: Algorithms based on Hamilton-Jacobi formulations," J. Comput. Phys., vol. 79, pp. 12-49, 1988.

[33] C. Loizou, C. Pattichis, M. Pantziaris, T. Tyllis, and A. Nicolaides, "Quality evaluation of ultrasound imaging in the carotid artery based on normalization and speckle reduction filtering," Med. Biol. Eng. Comput., vol. 44, pp. 414-426, 2006.

[34] T. J. Tegos, M. M. Sabetai, A. N. Nicolaides, T. S. Elatrozy, S. Dhanjil, and J. M. Stevens, "Patterns of brain computed tomography infarction and carotid plaque echogenicity," J. Vasc. Surg., vol. 33, no. 2, pp. 334-339, 2001.

[35] D. Mumford and J. Shah, "Optimal approximation by piecewise smooth functions and associated variational problems," Commun. Pure Appl. Math., vol. 42, pp. 577-685, 1989.

[36] T. Elatrozy, A. Nicolaides, T. Tegos, M. Griffin, and M. Sabetai, "The effect of b-mode ultrasonic image standardisation on the echodenisty of symptomatic and asymptomatic carotid bifurcation plaques," Int. Angiol., vol. 17, pp. 179-186, 1998.

[37] I. Wendelhag, Q. Liang, T. Gustavsson, and J. Wikstrand, "A new automated computerized analyzing system simplifies readings and reduces the variability in ultrasound measurement of intima-media thickness," Stroke, vol. 28, no. 11, pp. 2195-2200, 1997.

[38] D. J. Williams and M. Shah, "A fast algorithm for active contours and curvature estimation," Int. J. Graph., Vis. Image Process.: Image Understand., vol. 55, pp. 14-26, 1992.

[39] D. Huttenlocher, G. Klanderman, and W. Rucklidge, "Comparing images using the hausdorff distance," IEEE Trans. Pattern Anal. Mach. Intell., vol. 15, no. 9, pp. 850-863, Sep. 1993.

[40] J. S. Suri, R. M. Haralick, and F. H. Sheehan, "Greedy algorithm for error correction in automatically produced boundaries from low contrast ventriculograms," Pattern Anal. Appl., vol. 3, pp. 39-60, 2000.
[41] F. Destrempes, J. Meunier, M.-F. Giroux, G. Soulez, and G. Cloutier, "Segmentation in ultrasonic b-mode images of healthy carotid arteries using mixtures of nakagami distributions and stochastic optimization," IEEE Trans. Med. Imag., vol. 28, no. 2, pp. 215-229, Feb. 2009.

[42] F. Faita, V. Gemignani, E. Bianchini, C. Giannarelli, L. Ghiadoni, and M. Demi, "Real-time measurement system for evaluation of the carotid intima-media thickness with a robust edge operator," J. Ultrasound Med., vol. 27, no. 9, pp. 1353-1361, 2008.

[43] J. M. Bland and D. G. Altman, "Statistical methods for assessing agreement between two methods of clinical measurement," Lancet, pp. 307310, 1986.

[44] C. Schmidt and I. Wendelhag, "How can the variability in ultrasound measurement of intima-media thickness be reduced? studies of interobserver variability in carotid and femoral arteries," Clin. Physiol., vol. 19, pp. 45-55, 1999.

[45] A. Abdel-Dayem, M. El-Sakka, and A. Fenster, "Watershed segmentation for carotid artery ultrasound images," in Proc. 3rd ACS/IEEE Int. Conf. Comput. Syst. Appl., 2005, pp. 131-135.

[46] F. Molinari, G. Zeng, and J. Suri, "Intima-media thickness: Setting a standard for a completely automated method of ultrasound measurement," IEEE Trans. Ultrason., Ferroelectr. Freq. Control, vol. 57, no. 5, pp. 1112 1124, May 2010

Authors' photographs and biographies are not available at the time of publication. 